\title{
Angebornes Colobom der Iris und der innern Membranen des Auges.
}

Von

\author{
Dr. Albrecht Nagel.
}

\begin{abstract}
Anatomische Untersuchungen ${ }^{*}$ ) haben ergeben, dass in Fällen von angebornem Iriscolobom fast constant gleichzeitig Abnormitäten im Innern des Auges gefunden werden, welche gleich dem Irisdefect von einer Hemmung der fötalen Entwickelung abzuleiten sind. Die seither in der Literatur erwäbpien Fälle von Colobom der innern Augenbäute sind mit nur zwei mir bekannten Ausnahmen **) nur Gegenstand der pathologischen Ana-
\end{abstract}

*) v. Ammon, R. Wagner, Heyfelder, Gescheidt, Hannover, Arlt, Stellwag von Cariou.

*) Ein Fall von Stellwag (Zeitschr. d. Ges, der Wien. Aerzte 1856. No. 49. 50 wird unten noch erwähnt werden). v. Gräfe erwähnt eines Falles von Iriscolobom in einem mikrophthalmischen Auge (Archiv f. Ophth. II. 1.p. 239) bei dem indess die ophthalmoskopische Exploration wegen starken Nystagmus so schwierig und unvollkommen ausführbar war, dass zwar das Yorhandensein eines Choroidalefects aber nichts Näheres über denselben eonetativ werden konnte. Leider wird es noch oft genug versäumt. in solchen Fâllen den Augenspiegel cux Untersuchung der tieferen Theile des Auges zu benutzen und noch in allerneuester Zeit finden wir Fälle beschrieben, wo des ophthalmoskopischen Befundes nicht mit einem Worte Erwähnung geschieht. So finden sich in den Ophthalmic Hospital Reports des Letzten Jahres interessante Fälle ron Iriscolobom (Whice Cooper, Dixon, Streatefeild) obne dass 


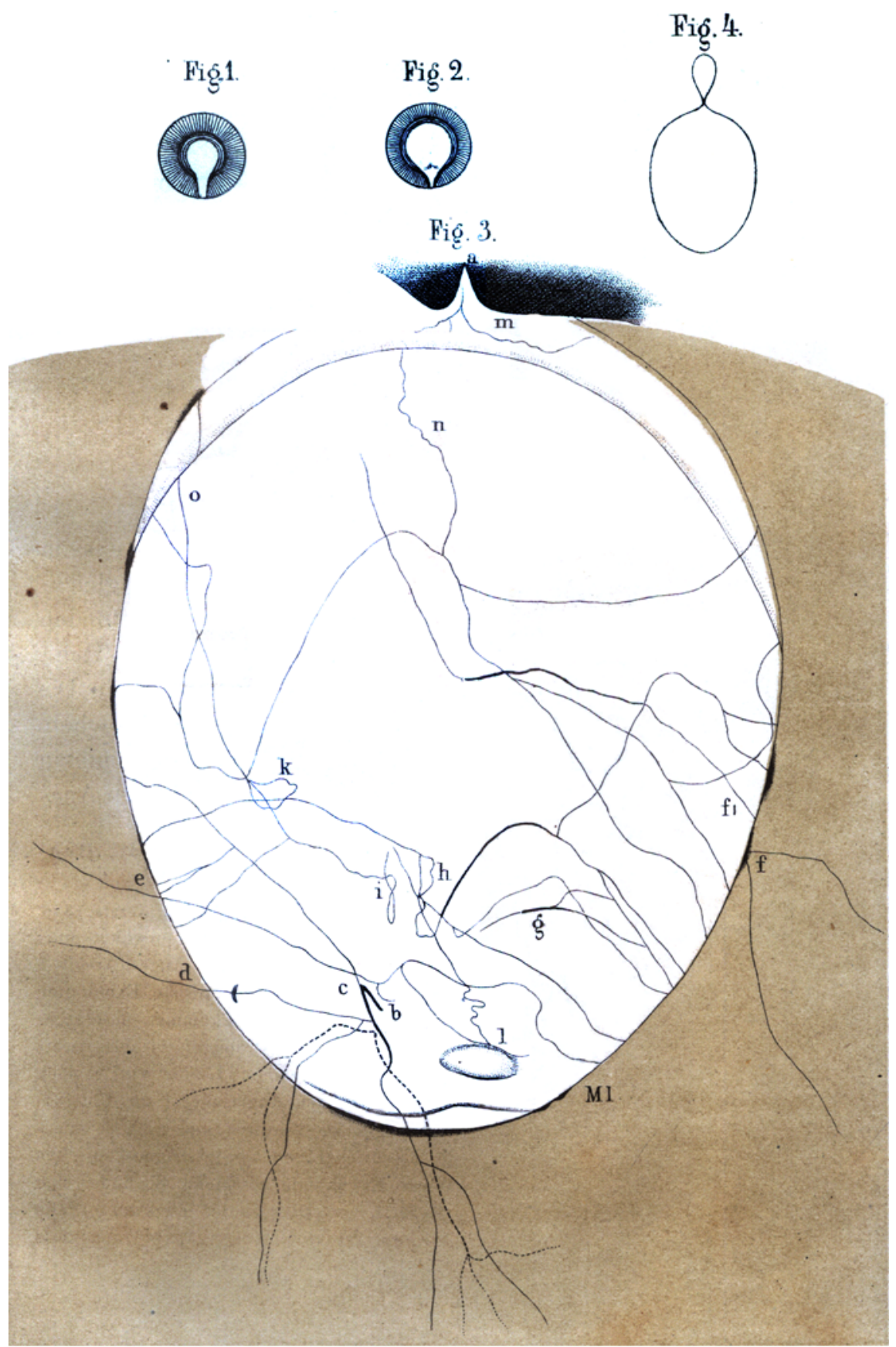


tomie gewesen. Da uns jetzt die ophthalmoskopische Untersuchung häufiger Gelegenheit bietet, uns intra vitam über den Zustand der inneren Membraneu zu unterrichten, so ist zu hoffen, dass manche weiterer Aufklärung bedürftige Punkte in der Geschichte dieser Missbildung nun um so lejchter ihre Erledigung fonden werden. Vergleichung genau beschriebener Fälle kann dabei von grossem Nutzen sein und so dürfte es nicht überfïssig erscheinen, wem ich mir splaube, einige Notizen über mehrere von mir beobachtete Fälle von Defect der inneren Membranen in diesem Archiv niederzulegen. In jedem der fünf Augen mit Iriscolobom, welche ich zu untersuchen Gelegenheit hatte, fand ich entsprechende Veränderungen im Innern des Auges; es bilden diese Fälle gewissermassen eine Scala der verschiedenen Grade der in Rede stehenden Bildungshemung; einer von ihnen (der zuletzt beschriebene, wo der Sehnerv noch ganz im Bereich des pathologischen Spaltes lag) bot einen so hohen Grad dar, wie ich ihn in der Zahl der bisher anatomisch beschriebenen Fälle nicht angegeben finde.

Ich erwähne die einzelnen Fälle in der Reihenfolge, wie sie die verschiedenen Grade repräsentiren, mit dem geringsten beginnend.

Ein Knabo mit nach unten gerichtetem Colobom mit dem Augenspiegel wird eine grosse, glänzend weisse, ziemlich excentrische Plaque gesehen, auf welcher einzelne rothe Gefässe sichtbar waren. Die Zeit der Beobachtang fiel in die erste Periode der Benutzung des

über die Betheiligung der iuneren Membranen etwas gesagt wäre. In einem Falle (Dixon) liess eine starke Beschränkung des Gesichtsfeldes nach oben (Objects placed abore the level of the eye were quite invisitle) einen Spalt der innern Membranen wohl mit Sicherheit voraussetzen; allein Dix on schrcibt dieselbe dem Drucke zn, welchen die verkalkte und nach unten dislocirte Linse anf den untern Theil der Retina ausiben soll (?). 
Augenspiegels und ich deutete den Befund damals als ein Exsudat; hege jetzt aber nicht den geringsten $Z$ weifel, dass es sich um einen circumscripten Choroidaldefect handelte, der in seiner Lage mit der Richtung des Irisspalts übereinstimmte d.h. sich in der VerJängerung desselben betand.

Ein zweiter Fall, welchen ich im Sommer 1857, leider nur einer kurzen und oherhächlichen Untersuchung unterwerfen konnte (olune künstliche Dilatation der Pupille) betraf Fräulein J. aus Br., eine Dame mit schwarzem Haar und dunkelbrauner Iris. Beide Augen zeigten ein nach mten und etwas nach innen gerichtetes completes Iriscolobom; die Pupillen waren lebhaft beweglich; das normale Pupillargebiet ein wenig excentrisch, in der Richtung des Coloborus dem Ciliarrande etwas näher gerïckt. Form und Grösse der verlängerten Pupille in beiden Augen durchaus gleich und symmetrisch. Die ophthalmuskopische Exploration orgab in beiden Augen einen im unteren Theile des Augengrundes belegenen, grossen Choroidaldefect; ein so weit es sich verfolgen liess, scharf ungränztes, glänzend weisses Halboval, dessen längere Axe in dem dor Richtung des Iriscoloboms entsprechenden Meridian des Bulbus lag und dessen abgerundetes, tem Sehnrryen zugekehrtes Ende von diesem um etwa das Anderthalbfache des Durchmessers der Papille entfernt war. Ob sich der Defect jenseits des Bulhusäquators und in den Ciliarkörper hincin fortsetzte und mit dem Iriscolobom in directen Zusammenhange stand, konnte der engen Pupille wegen nicht ermittelt werden. Das glänzendweisse sehnenartige Aussehen der Figur im Augengrunde rührt unzweifelhati von gänzlichem Fehlen der Choroidea (wenigstens des Gefäss- und Pigmentstratums) und dem Freiliegen der Silerotica her. Die Netzhaut war, soweit die Untersuchung sich erstrecken konnte, 
in ihrer Continuität ununterbrochen; ihre Gefässe liessen sich in gewöhnlichem Verlaufe mit ihren Verzweigungen auf die Defect-Stelle verfolgen und markirten sich deutlich auf dem hellglänzendem Grunde. Neben denselben waren hier jedoch noch einzelne feine hellrothe Gefässe sichtbar, welche ihrem Verlaufe nach nicht Netzhautgefässe sein konnten, sondern einem darunter liegenden Stratum angehören mussten. (Vasa ciliaria post.) Der übrige Augengrund bot keine von den normalen wesentlich abweichenden Charactere dar, er war namentlich in der Umgebung des Opticus pigmentarm, so dass man den Verlauf der gedämpft rothen Choroidalgefässe deutlich erkennen und bis an die Grenze des pathologischen Spaltes vertolgen konnte. An der Grenze der weissen Figur wurde stellweise Anhäufung und Unregelmässigkeit des Pigments bemerkt. In der Nachbarschaft des Sehnerven zeigt, besonders auf einem Auge die Choroidea eine sichelförmige, an den Sehnerven sich anschliessende atropische Stelle mit kleinen Pigmentanomalien, wie bei den gewöhnlich in Begleitung der Myopie vorkommenden leichten Scleralectasien. Das Sebvermügen konnte nicht so genau geprüft werden, als e's wünschenswerth gewesen wäre. Doch ging aus der kurzen Untersuchung hervor, dass keine irgend wesentliche Störung vurhanden war. Jedenfalls war ein erheblicher Defect im Gesichtsfelde, der pathologischen Region des Augengrundes entsprechend, nicht vorhanden.*) Von Interesse war es dass, die Accommodation vollkommen gut war (oder wie ich bei dem Mangel einer Messung lieber sagen will: keine merkliche Beschränkung zeigte); entgegenstehend den vielfachen Behauptungen von nachtheiligem Einflusse der Irisdefecte

*) Fs ist bekannt, dass auch bei acquisiten Choroidalatrophieen die atrophische Stellen bedeckende Retina in ihrer Function ungestört sein kann. 
auf das Accommodationsvermügen, die übrigens durch vielfache Erfahrungen über die auf operativem Wege erzeugten Irisdefecte zur Genüge widerlegt sind.

Im Dezember 1857 hatte ich Gelegenheit im Danziger Krankenhause ein 9jähriges Mädchen rnit angebornen Iriscolobom zu sehen. Auch in diesem Falle bin ich, da einer Aufforderung zu wiederholter Vorstellung nicht Folge gegeben wurde, auf das Ergebniss einer einzigen flüchtigen Untersuchung beschränkt.

A. Gr. aus der Umgegend von Danzig hat ein ganz mikrophthalmisches rechtes Auge von der Grössr einer kleinen Haseluuss mit getrübter Cornea; auf dem andern, Auge, dem linken*), - mit dem sie noch so viel sieht, um sich zu führen, bei unsicherer Fixation in geringer Entfernung. Finger z11 zählen und von den Jägerschen Probe-Streifen bis 55 zu unterscheiden ein nach unten gerichtetes completes Colobom der Iris. Die Untersuchung der innern Membranen gab ein ganz ähnliches Resultat, wie in dem zuvor erwähnten Falle, daher ich die Beschreibung nicht in extenso wiederhole, sondern nur die Abweichungen erwähne. Ein eben solcher, nur etwas breiterer der Lage nach wiederum dem Defect in der Iris correspondirender Mangel der Choroidea konnte nach der Peripherie hin bis über den Aequator des Bulbus hinaus verfolgt werden und war andrerseits von dem Sehnerven dureh eine Zone getrennt, in welcher sich wieder eine gestreckt inselförmige der Choroidea entbehrende Stelle befand. Auch in unmittelbarer Umgebung des Sehnerven ist die Pigmentschicht der Choroidea efwas atrophirt, so dass derselbe unregelmässige, zerrissene Ränder zeigt; überhaupt finden sich im Augengrunde mehrfache Pigmentanomalien,

*) Die statistischen Zusammenstellungen ergeben, dass das congretinaie Iriscolobom ïberwiegend häufiger auf dem linken Auge beobrchtet wird. 
und es war von besonderem Interesse auch im Areal des Defects in der Gegend des Aequators Spuren von Pigment zu fuden. Die Netzhaut ging wie aus dem Verlauf der Gefässe zn schlirssen war, sowohl uber den grossen Defect als über den kleinen inselförmigen hinweg. Die parallaktischen Verschiebungen derselben an den Räudern des Choroidalspalts hewiesen, dass die. von der Choroidea nntblösste Scleralparthie staphylomatös ausgebuchtet war.

Den höchsten Grad endich des Choroidalcoloboms, höhr als in irgend einem bisher beschriebenen Falle beobachtete ich im Dezember 1856 und es scheint mir dieser in mebrfacher Beziehung interessante Fall wohl einer etwas ausftihrlicheren Beschreihung werth.

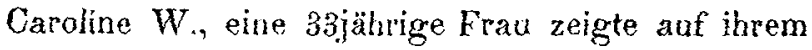
rechten stark amblyopischen Auge ein beinahe gerade nach unten gerichtetes Iriscolobom. Mit diesem Auge wurden bei etwas unsicherer Fixation Finger auf einige Fuss Entfernung gezählt, Streifen No. 20-25 in den Jägerschen Proben erkannt. Das Gesichtsfeld zeigte eine starke Beschränkming nach oben. In der Mittellinie fand über die Horizontale hisaus keine Perception mehr statt, wäbrond dieselbe an beiden Seiten noch eine ziemliche Strecke nach oben uber die Horizontale hinaus ging. (Durchaus in Vebereinstimmung mit der ophthalmoskopisch eruirten Veränderung im Augengrunde). Das linke Auge zeigte keine Andeutung ron Golobom, war normal bis auf leichte Presbyopie*) and einen geringen Grad von Hemeralopie (der seit Ku'zem wohl im Zusammenhang mit einer, durch Ueber-

* So sebrieb ich damals; genauer bezeichnet: Abrïckung des Bereichs der ausdavernden Accommodation, da für dauernde Besehäfigung in der Näbe Convexgläser erforderlich waren. Nach den neuesten, dureh Oonders eingeführten Anschauungen, wäre diesc, übrigens sehr gewöhnliche Affection als facultative Hyperopie ou bezeichnen. 
anstrengung der Accommodation erzeugten Netzhauthyperämie bestand).

Der rechte Bulbus war in sehr in geringem Grade mikrophthalmisch. Obgleich er beim ersten Anblick ein wenig kleiner erschien, als der linke, so ergaben sich doch durch die Messung die Höhen- und Quer-Durchmesser beider Hornhäute als kaum merklich verschieden. Der Widerspruch erklärte sich bei genauerer Betrachtung dadurch, dass neben kleinen Unterschieden in den Dimensionen der Lidspalte die rechte Horuhaut, obgleich in Höhen- und Quer-Durchmesser der linken gleich, dennoch ein etwas kleineres Areal einnahm als die linke, indem bei ersterer der untere Halbkreis ihrer Peripherie von den Seiten ein wenig zusammengedrückt war, und so die Hornhaut ein leicht birnförmiges Ansehen erhielt.*) Form and Consistenz des Bulbus zeigen keine Abweichung von der Norm. Die vordero Kammer war augenscheinlich rechts flacher als links, indem die rechte Iris ein wenig mehr nach vorn gedrängt war**); die Hornhautkrümmung dagegen schien auf beiden Seiten gleich zu sein, wenigstens konnte mit freiem Auge ein Unterschied in der Grösse der Reflexbilder nicht erkannt werden. Die Iris des normalen linken Auges war dunkelblaugrau, dagegen die des rechten hell-gelblich-grau, in den unteren, dem Colobom benachbarten Parthieen noch heller als in den oberen. Auch die Textur der Iris war nicht ganz normal, das Faserwerk namentlich an einzeinen inselartigen Stellen

*) Die Maasse waren in beiden Augen folgende:

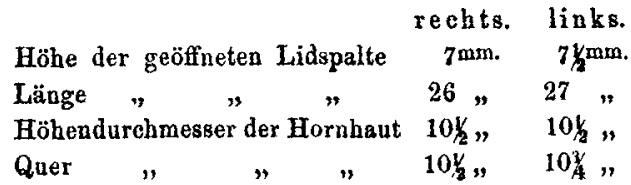

**) Ioh bemerke dies, da der myopische Bau des rechten Anges das Gegentheil erwarten liess. 
dicht scharf ausgeprägt, etwas verwischt. Das nach unten nur um wenige Grade nach innen gerichtete Colobom der Iris war ein vollständiges; indem der Spalt mit nach unten etwas convergirenden Rändern bis an den Ciliarrand der Iris reichte. Die dadurch gebildete vergrösserte Pupille hatte die in Fig. 1 bezeichnete nach unten verjüngte Form, an der man die eigentliche $\mathrm{Pu}$ pille von dem pathologischen Spalt durch einen kleinen Absatz, einen vorspringenden Winkel getrennt erkennen konnte. An diesem die Grenze der centralen und der accessorischen Pupille bezeichnenden Absatz hört der im Umfang der centralen Pupille in gewöhnlicher Weise entwickelte imnere Kreis der Iris auf und :die Spaltränder, welche je weiter nach unten um so deutlicher nach vorne ein wenig umgeschlagen waren*), zeigten von einem inneren Kreise keine Spur, sondern einen feinen, schwärzlichen, durch Ueberragen der Pigmentschicht gebildeten Rand. Das Gebiet der centralen Pupille war, wie aus den unten angeführten Maassen des Näheren hervorgeht, um Einiges nach unten verrückt. Die Pupille erschien vollkommen schwarz und zeigte gegen Lickt einen mässigen Grad von Beweglichkeit; die Verengerung ging in der Weise vor sich, dass sowohl der senkrechte als der Querdurchmesser der Gesammtpupille sich verkleinerte; dabei war eine Veränderung in Form und Grösse sowohl in der centratralen als in der accessorischen Pupille wahrzunehmen. Auf Instillation einer Lösung von schwefelsaurem Atropin dilatirte sich die Pupille schnell und ziemlich bedeutend und nahm dann die in Fig. 2 widergegebene

*) Dasselbe fand Stellwag Ophthalmologie II p. 178; der auch durch das Mikroskop feststellte, dass die Sphincterenden durch gradlinigte Faserbïndel, welche die umgesehlagenen Ränder des Coloboms bildeten, mit dem Ciliarmuskel in Verbindung standen - daher auch die Dislocation der centralen Pupille. 
Gestalt an; die Erweiterung geschah in allen Durchmessern, am schwächsten am Ciliarrande des Coloboms, wo sie nur ${ }^{4 / 10} \mathrm{~mm}$. betrug.*) - Die Ciliarfortsätze, deren Firsten bei dem Mangel des sie im normalen Auge verdeckenden Iristheils bei schief auffallender Focalbeleuchtung sichtbar sein mussten ${ }^{* *}$ ) fehlten in der Mitte gänzlich; nur zu beiden Seiten des Spaltes konnten die benachbarten stark abgeschrägten Processus gesehen werden (cf. Fig. 2).

Die völlig durchsichtige Linse zeigte, sowohl bei Fokalbeleuchtung als bei durchfillendem Licht mit dem Augenspiegel untersucht, an ihrer Peripherie gerade im Bereich des Iriscoloboms eine seichte Einkerbung (cf. Fig. 2), auch war hier der Linsenrand etwas dunkler contourirt als er es im normalen Zustande zu sein pflegt. Unterhalb der Linsenperipherie sah man zwischen dieser und der Sclera hindurch mit Hülfe des Augenspiegels den erleuchteten Augengrund ohne die geringste Trübung; es konnte nicht ausgemacht werden, ob an

*) Die Messung der Pupille vor und nack der Dilatation ergab folgende Zahlen, die auf die schciubaren Digtancen, als Sehnen der Hornhautoberfläche gemessen, zu bezichen sind.

Höhendurchmesser der Pupille incl. Colobom Grösster Querdurchmesser der Pupille. . . Querdurchmesser am Anfange des Coloboms (beim Aufhören des inneren Iriskreises). Kleinster Querdurchmesser dern Hornhautrande entsprechend . . . . . . . Entfernung des vorspringenden Winkels des Pupillarrandes rom Giliarrande. . . .

\begin{tabular}{|c|c|}
\hline $\begin{array}{l}\text { Vorder Dila- } \\
\text { tationbejuâs. } \\
\text { sigem Licht- } \\
\text { einfall }\end{array}$ & $\begin{array}{c}\text { Nachde } \\
\text { Dila- } \\
\text { tation }\end{array}$ \\
\hline $64 \mathrm{~mm}$ & $8^{\mathrm{mm}}$. \\
\hline $5 "$ & 6, \\
\hline & $3 \%$, \\
\hline $2 y_{2}$, & 2,9, \\
\hline & $1 / 3$ \\
\hline
\end{tabular}

**) In Augen mit künstlichem Colobom, wo durch eine Iridectomie die Iris bis hart an ihre Ciliarinsertion entfernt ist, sieht man bei schiefer Fokalbeleuchtung jederzeit aufs Deutlicbste die Firsten der Proc. ciliares als einen bräunlichen mit seichten (meridionalen) Einkerbungen versehenen Wulst. 
dirser Stelle die Zomula Zinnii fehlte oder in normaler Durchsichtigkeit vorhanden war.

Die ophthalmoshopische Untersuchung zeigte sofort anflallende Anomalion. Brim arsten Blick gewahrte man inmitten des die gewöhnliche rothe Farbe zeigenden Augengrundes rin grosses. glänzend weisses, von sparsamen Gefässen durchzogrenes Feld (‘f. Fig. 3), das -ich nach genaurer lingrenzung als ein von seiner Umgebung äberall scharl' geschiedener nach dem Centrum des Augengrindes lin algerunder Sector, der als Kreis projicirt gedachten inneren $W$ and des $A$ uges erwies. Im umgekohrten IBilde ciel dieser Sector mitten in dessen oberen Theil, es gehörte also die entsprechende Veränderung der Mitte der untoren Hemisphäre des Bulbus an und es lag die Vermuthung nabe, dass die Anomalie mit dem Iriscolobom in Verbindung stele, was sich bei weiterer Untersuchung bestätigte. Die divergirend nach der Peripherie auseinanderfahrenden Grenzen des weissen Feldes liessen mich anfangs annehmen, dasselbo erstecke sich bis an den Ciliarrand der Iris und es bestehe ein offener Zusammenhang zwischen dem Defect der Iris und dem Defect der inneren Membran, doch gelang es mir später bei den durch den Irisspalt und den durch glänzendeu Scleralretlex begünstigten Beleuchtungsverhältnissen noch die jenseits des Augenäpuators gelegenen Theile bis zum Giliarkörper zu sehen und da fand sich denn, dass die anfangs radinatig divergirenden Grenzen des weissen Feldes convergirten und sich schliesslich zu einer Rundung vereinigten, welche ein regelmässiges Oval von beträ̈htlicher Ausdehnung abschloss. IDiese oberste*) Grenze bildete nicht mehr der rothe Augengrund, sondern ein tice schwarzer, mit dem Augenäquator parallel

*) Die Ortsbezeichnungen beziehen sich auf das ungekehrte Bild, wie es in Fig. 3 darirustellt ist. 
gehender Streifen, der sich nur mit grösster Mühe leichter nach der rechten als nach der linken Seite verfolgen liess. Ziemlich in der Mitte dieses schwarzen Streifens machte sich ein kleiner mit seiner Spitze aufwärts gerichteter Ausschnitt (Fig. 3) bemerklich, der eine Fortsetzung des weissen Feldes bildete, so dass das letztere einen spitzen Ansatz an seine abgernndete Contour zeigte.

Die Deutung dieses Befundes war in den Hauptpunkten einfach genug. Der weiss reflectirende $A b$ schnitt des Augengrundes konnte nur von der durch Mangel der Choroidea frei liegenilen Sclerotica herrühren. Die Lage des Defects war derart, dass derselbe offenbar mit dem Bildungsfehler in der Iris in Zusammenhang stehen musste, jedoch bestand nicht, wie ich erwartete, eine einfache Continuität zwischen beiden Defecten, sondern der zwischen Iris und Choroidea liegende Theil der Uvea, der Ciliarkörper mit seinen Fortsätzen und dem Tensor schien, wenn auch an der Anomalie betheiligt, so doch nur in geringem Grade defect zu sein. Es bedarf nicht des Beweises, dass der so eben beschriebene schwarze Streifen an der oberen Grenze des Choroidaldefects den auf die Ora serrata folgenden flachen Theil des Ciliarkörpers, und dass der beschriebene Ausschnitt einen theilweisen Spalt in demselben darstellt; einen Spalt mit abgeschrägten Rändern ganz in derselben Weise, wie in umgekehrter Richtung der Spalt der Iris sich in den vorderen Theil des Ciliarkörpers fortsetzte. Ueber den Zustand des mittleren Theils des Ciliarkörpers, insbesondere des Ciliarmuskels konnte natürlich durch die Beobachtung nichts ermittelt werden.*) Da jedoch der ganze übrige

*) Nach meiner Schätzung konnte die Breite der Zone des Ciliarkörpers, welche sowohl der Untersuchung von aussen mit Focalbeleuchtung als von innen mit dem Ophthalmoskop entzogen blieb nicht 
Befund mit den anatomischen Befunden in ähnlichen Fällen genau übereinstimmt, so wird man mit Wahrscheinlichkeit schliessen dürfen, dass hier analog wie dort die beiden gegeneinander gekehrten zugespitzten spaltförmigen Defecte durch eine Raphe in dem noch übrigen mittleren der Beobachtung unzugänglichen Theile des Ciliarkörpers verbunden seien. Die schematische Figur (Fig. 4) bezeichnet - den Uvealabschnitt in einer Ebene ausgebreitet gedacht - die Form, welche der gesammte pathologische Defect gehabt haben muss.

Der übrige Augengrund bot ausser etwas stärkerer unregelmässiger Pigmententwickelung längs des Randes des Choroidalcoloboms keine auffällige Abnormität dar; er zeigte die gewöhnliche Farbe in ziemlich dunkler Nüance; Netzhautgefässe waren in normaler Weise vertheilt; der Verlauf der Choroidalgefässe konnte an den Ungleichheiten der Pigmentschichten stellenweise erkannt werden. Von der Sehnervenscheibe war nichts zu sehen; sie musste, wie die Betrachtung der räumlichen Verhältnisse lehrte, innerhalb des weissen, pigmentlosen Feldes liegen, und der Verlauf einiger Gefässe gab auch sichere Anhaltspunkte für die Localisation (cf. weiter unten). Nichts desto weniger vermochte ich die Eintrittsstelle durch kein anderweitiges Merkmal von der umgebenden Sclerotica zu unterscheiden; keine Spur von einer Contour, kein Unterschied in Farbe oder Glanz; der abnorme Verlauf der Central-

mehr als $1-1 \frac{1}{2}$ Linie betragen. Nur dann würden sie breiter sein, wenn der von mir gesehene flache Theil des Ciliarkörpers sich nicht in seiner normalen Lage befunden hat, sondern, wie einige Sectionen colobomatöser Augen ergeben haben, nach unten dislocirt war - ein Punkt über den ich damals keinen Aufschluss gewinnen konnte. Leider habe ich in jüngster Zeit die Beobachtung nicht wiederholen können, es würden sich bei dem schnellen Fortschritt der ophthalmoskopischen Untersuchungsmethode vielleicht manche befriedigendere Aufschlüsse ergeben haben. 
gefässe (Fig. $3 b$ und $c$ ) und der Mangel der normalen Durchscheinendheit der Nervensubstanz und der Sichtbarkeit der Lamina cribrosa dentete auf eine Abnormität in der Bildung des Opticus. Ebensowenig als die Papilla optici markirte sich in irgend merklicher Weise die Stelle des directen Sehens; welche ausserhalb des Choroidaldefects, dessen Rand ganz nahe (an der mit $M l$ bezeichneten Strlle der Fig. 3) gesucht werden musste. Von den gewöhnlichen Kennzeichen der Macula lutea war nichts zu sohen.

Der Verlauf der Gefässe im Bereich des Choroidaldefects war ein von den normalen Verbältnissen gänzlich abweichender; Jas studium desselben musste fïr die Beurtheilung der Natur der die Sclerotica bedeckenden Membranen wichtig sein. Die Fig. 3 liefert eine, so weit meine greringe Unbung in den zeichnenden Künsten erlauble, möglichst treue Skizze der Gefüssverzweigung, mit Weglassung einiger ganz feiner Zweige. Im Allgemeinen stimmten die auf der Sclerotica sichtharen Gefässe in ihrem Verlauf weder mit der gewöhnlichen Vertheilung der Netzhaut- noch der Choroidalgefässe überein. Nur im untersten Theile der weissen Figur characterisirten sich einige Gefüsse deutlich als Netzhautgefässe. Es liefen hier in der gewöhnlichen Weise Arterien und Venen, die in den rothen Augengrund leicht zu verfolgen waren, neben einander; einige grössere Stämme vereinigten sich und brachen plützlich ab (in $b$ Fig. 3 ) an einer Stelle, die auch der Lage nach für die Papilla 1 . optici gehalten werden musste. Dicht daneben machte ein mit jenem confluirendes Gefäss ( $c$ Fig. 3 ) einen sohr spitzen Winkel und verschwand dann plötzlich - eine Configuration, wie sie auf dem normalen Opticus niemals gefunden wird. Noch einige andere Netzhautgefässe des rothen Augengrundes liessen sich in den Defect hinein verfolgen (z. B. 
das Geläss e Fig. 3), einige nur bei gewissen Stellungen (wie das Gefäss $f . f_{1}$ ), so dass sich auf eine Veränderung des Niveaus und zwar eine Ausbuchtung der der Choroidea beraubten Stelle der Bulbuswand schliessen liess. Bemerkenswerth war es, dass diese eben erwähnten Netzhautgefässe keine directe Communication mit der Austrittsstelle der Centralgefässe zeigten, dass also die Rotina ihr Blut aus verschiedenen Quellon bezog. Die Mehrzahl der auf der Sclerotica liegenden Gefässe liess sich, ohne Zusammenhang mit Netzhautgefässen, nur bis an den Rand des Choroidalspalts verfolgen, ging also, wie mit Sicherheit anzunehmen ist, in das von der Pigmentschicht bedeckto Gefässlagrer der Choroidea über. Diese Gefässe, die übrigens mit Ausnahme der oben erwähnten wenigen Retinal-Venenstämme im untern Theil (diese sind in der Figur dureh Punkte bezeichnel) die hellrothe Farbe der Arterien zeigten, waren von verschiedener Stärke; einige vou bedeutenderem Caliber als Netzhautgefässe zu haben pllegen. Der Verlauf war ein ganz unregelmässigur; sie anastomosirten mehrfach untereinander und stellenweise auch mit dea Netzhautgefässen; einige nahmen einen ganz eigenthümlich gewundenen in sich selbst zurücklaufenden Gang (h. i. k. l. in Fig. 3). kinige brachen plötzlich ab, durchbohrten also die Sclerotica; ein Gefäss $(g)$ setzte sich nach dem Verschwinden der rothen Farbe als ein bräunlicher mehr und mehr undeutlicher werdender Streifon eine Strecke weit fort. Ich zweille nicht, dass es hiemit dieselbe Bewandniss hat, wie in albinotischen Augen mit den bräunlichen Punkten oder Streifen an der Durchtrittsstelle der Choroidalgefüsse durch die Sclera, dass nämlich der bräunliche Streifen den Lauf des die Sclera schräg durchbohrenden Gefässes bezeichnet. Diese zuletzt beschriebenen Blutgefässe können füglich nur als 
hintere kurze Ciliararterien angesprochen werden. $\mathrm{Ob}$ dieselben frei über die Sclera hinzogen, oder in einer eigenen, zwischen Netzhaut und Sclera befindlichen Membran verliefen, welche vielleicht eine rudimentäre Entwickelung des Choroidalstromas darstellt; ob die Communication des Retinal- und Ciliargefüsssystems in einer Verwachsung der Netzhaut mit Sclera oder einer solchen Zwischenmembran ihren Grund hatte, ob endlich die Netzhautelemente sich in normalem, oder, was wahrscheinlicher, in verändertem Zustande befanden, diese Fragen konnten auf ophthalmoskopischem Wege nicht entschieden werden. Ich komme auf diese Punkte noch weiter unten bei der Vergleichung des ophthalmoskopischen Befundes mit anatomischen Befunden mit einigen Worten zurück.

Ueber die Beschaffenheit der von der Sclera gebildeten weissen Figur ist wenig hinzuzufügen. Dieselbe bildete ein ziemlich gleichförmig weisses stark refectirendes Feld; der Glanz war nicht überall gleich stark; eine vorzugsweise stark glänzende Stelle befand sich ziemlich in der Mitte der Figur; andere Stellen zeigten eine mehr fasrige Structur; so besonders das rechte Seitentheil. Hin und wieder, namentlich an den Rändern waren einzelne bräunlich pigmentirte Stellen, theils in grösseren Streifen und Inseln, theils als feine Punktirung. Ich möchte, die kleinen Ungleichheiten im Aussehen der Sclerotica auf die verschieden feste und innige Adhärenz, Verwachsung derselben mit der sie bedeckenden Membran beziehen; sei diese letztere nun allein die Netzhaut oder ein die Choroidea vertretendes Zwischenhäutchen.

Es ist bereits erwähnt worden, dass die Bulbuswand im Bereich des Choroidalspaltes nach hinten ausgebuchtet war, also ein Scarpa'sches Scleralstaphylom darstellte. Es konnte dies ausser aus den Gefässver- 
schiebungen am Rande der Figur auch durch die verschiene Stärke der negativen Gläser festgestellt werden, welche zur Beobachtung verschiedener Parthien des Augengrundes im aufrechten Bilde erforderlich waren. Zur Beobachtung der am tiefsten gelegenen mittleren Parthie brauchte ein normales Auge ein Glas von etwa 5 Zoll negativer Brennweite, während die Randparthieen und mehr noch der übrige Augengrund mit viel schwächeren Gläsern deutliche Bilder lieferten. Die Ectasirung schien nicht überall gleichmässig zu sein. Der Uebèrgang von dem Rande zur Mitte schien am untern Theil in der Gegend der Macula und des Opticus flacher und allmählicher zu sein als in der Mitte, wo die Gefässverschiebungen bei verschiedenen Stellungen sehr bedeutend waren. Ein besonderes Interesse gewährte noch der peripherische, der Ora serrata zunächst gelegene Theil des Colohoms; der freilich seiner Lage wegen der Beobachtung grosse Shwierigkeiten bot. Hier schien der Rand der Seleralectasie sehr steil zu sein, der Ciliarkörper prominirte relativ stark, indem er sich leicht äber das ihm zunächst gelegene Geräss $m$ (Fig. 3) verschob. Ganz nahe der Ora serrata und längs :derselben erstreckte sich eine schmale, durch eine schwache Contour begrenzte Zone an deren Grenze die dort sichtbaren Gefässe eine Aenderung in ihrem Lauf erlitten. Das Gefäss $n$ konnte gar nicht weiter verfolgt werden; ist aber wahrscheinlich die Fortsetzung des Gefässes $m$; das Gefäss $O$ dagegen konnte mit starker Verschiebung weiter verfolgt werden und bei einer gewissen Stellung war der Verbindungstheil beider Gefässe in perspectivischer Verkürzung sichtbar (wie die Figur bezeichnet). Ich lasse es dahingestellt, ob diese Irregularitäten nur der optische Ausdruck des steil aufsteigenden Randes des Scleralstaphyloms waren oder ob sie vielleicht 
durch Unregelmässigkeiten in der Hyaloidea oder im Glaskörper veranlasst wurden.

Die Vergleichung des mit einer Ausführlichkeit, welcher das Interesse an der noch jungen ophthalmoskopischen Technik zur Eutschuldigung dienen möge beschriebenen ophthalmoskopischen Bnfundes mit anatomischen Befunden in colobomatïsen Augen, erkläri die meisten Details der Beobachtung. Einiger Punkte möchte ich noch speciell Erwähnung thun. Es ist sicher, dass wir es hier mit einem Stehenbleiben auf einer normalen Entwicklungsstufe zu thun hahen; nicht mit dem Resultate eines fötalen Krankheitsprozesses im engeren Sinne. - Die in Begleitung des Irisspaltes vorkommenden angebornen Anomalien der inneren Theile des Auges lassen sich sämmtlich auf dic unvollkommene Schliessung der fütalen Augenspalte zurückführen. Es scheint, dass die verschiedenen Formen und Combinationen dieser Anomalien als verschiedrne Grade einer und der'selben Entwickelungshemmung zu betrachten sind und eine zusammenhängende Reihe bilden - deren niedrigster Grad das einfache incomplete Irisculobom ist, deren höchster Grad der durch alle Theile der Uvea und die Netzhaut gehende spaltförmige Defect, verbunden mit staphylomatöser Ausbuchtung der Selera und mit gewissen Auomalien des Glaskürpers, der Linse und deren Befestigungen. Da die Iris ein secundäres Organ ist, welches erst spät durch Anschiessen ihrer Substanz an den vorderen Rand der Choroidea sich entwickelt, so ist ihre Bildung ganz von den Zuständen des Mutterbodens um diese Zeit abhängig und Störungen in diesem müssen sich in ihr unfehlbar reflectiren. So ist es erklärlich, dass das Colobom der Iris, die unvollkommene Spaltschliessung in Choroidea und Ciliarkörper stets begleitet; dass aber der Grad der inneren Abnormitäten, die bei gleichem Irisdefect gefunden 
werden, sehr verschieden sein kann. Der Choroidalspalt kann sich noch in späterer Zeit ganz oder theilweise schliessen, der Irisspalt nicht, wenn nicht die Schliessung an seiner Ursprungsstelle stattgefunden hat. - Es fragt sich, wio dio Entstehung des Scleralstaphyloms zu crklären ist. Ich lasse es unentschieden, ob die von v. Ammon in einer bestimmten Periode des Fötallebens constant beobachtete Protuberantia scleralis posterior*) damit im Zusammenhange steht. Jedenfalls scheint es mir wahrscheinlich, dass dem intraocularen Druck bei der Bildung dieses Staphyloms eine aetiologische Bedeutung beizumessen ist. Die Schliessung des fötalen Augenspaltes geschieht in der Sclera früher als in der Choroidea. Die Sclera ist auf früher Entwicklungsstufe noch sehr dünn und nachgiebig und insbesondere ist die den Verschluss des fötalen Augenspalts bildende Parthic anlangs noch sehr dünn. Bleibt nun die Vereinigung der Spaltränder der Choroidea (und Netzhaut) aus, so kann die Bulbuswand an dieser Stelle nicht die normalo Resistenz bieten und bei dem stärkeren Ausfüllen der Bubushöhle durch weitere Entwickclung ihrer Contenta und durch den grösser werdenden Blutdruck wird der zunehmende Druck die Spaltstelle als die schwächste Stelle der Bulbuswand vortreiben, so lange bis die Spannung sich mit dem Drucke ins Gleichgewicht gesetzt hat. Möglich, ja wahrscheinlich, dass hiedureh oft der Spalt grösser wird, als er ursprünglich war; möglich, dass gerade durch diese Wirkungsweise des intraocularen Druckes die spätere Vereinigung des Spaltrandes erschwert oder unmöglich gemacht wird, während die weiter nach vorn gelegenen Theile der Choroidea über denen die Sclera schon früher geschlossen war in ihrer Entwicklung weniger gestört

*) Archiv f. Ophth. IV, 1, pag. 40 und pag. 187. 
werden. So erklärt sich dann, dass der durch die ganze Uvea gehende Spalt im hinteren Theile der Choroidea am grössten ist, dass er dagegen im Ciliarkörper am unbedeutendsten ist, oft nur als Raphe mit einiger Dislocation, weil hier, selbst nachdem schon die Iris gebildet ist, eine nachträgliche Vereinigung der Spalte am wenigsten gehindert ist.

Am wenigsten aufgeklärt ist bis jetzt das Verhalten der Retina in colobomatösen Augen und in dieser Beziehung ist der letzte der oben beschriebenen Fälle von Interesse. In einigen Fällen fand man die Netzhaut diverticelartig in das Staphylom sich fortsetzend, und wenn auch in ihren Eigenschaften wesentlich alterirt, bildete sie doch ein Continuum und war nicht gespalten. In anderen Fällen zeigten sie in ihrem peripherischen Theile einen spaltartigen Defect, während sie über den dem Sehnerven zunächst gelegenen Theile des Scleralstaphyloms sich brückenartig hinüberspannte.*) In noch anderen Fällen endlich fehlte sie im Bereich des Staphyloms ganz und ihre Ränder hingen mit den Rändern des Choroidaldetects eng zusammen, während dann die innere Fläche der ectasirten Scleralparthie von einer dünneu, bindegewebigen, sparsame Gefässe enthaltenden $\mathrm{Zwischenmembran}$ ausgekleidet war.**) In dem vorliegenden Falle giebt der beschriebene Gefässverlauf Anhaltspunkte zur Beurtheilung der das Staphylom auskleidenden Membranen. Es geht aus demselben hervor, dass die Spaltstelle keineswegs der Retina entbehrte, dass diese aber ebensowenig von normaler Beschaffenheit sein konnte; - dass sie am meisten normal am centralen Ende des Coloboms war; am we-

*) Dies beobachtete Stellwag l. c. auch ophthalmoskopisch.

**) Diese Zwischennembran fand Arlt an den Ränden sowohl mit der Netzhaut als mit der Choroidea unzertrennlich vereinigt; mit beiden ein Continuum bildend. 
nigsten normal, vielleicht ganz fehlend am ciliaren Ende desselben. Wahrscheinlich war es ferner, dass der ganze ausgebuchtete Scleraltheil ausser von der Retina noch von einem dünnen durchsichtigen Häutchen bedeckt war, das von Vasa ciliaria post. durchzogen war, welche in die benachbarte Choroidea übergingen, aber auch mit Netzhautgefässen anastomosirten. Hiernach ist es mir am wahrscheinlichsten, dass Zwischenmembran uud Netzhaut (erstere vermuthlich mit directem Uebergange in das Choroidalstroma) beinahe der ganzen Ausdehnung nach zu einer einzigen Membran verschmolzen waren, welche in ihrem dem Opticus benachbarten Theile die Netzhantelemente und deren Gefässe noch in reichlicherer Menge enthält, während sie nach der Peripherie zu ärmer an diesen wird; sie vielleicht ganz verliert und allein aus Bindegewebe besteht; vermuthlich auch der ganzen Ausdehnung nach mit der Sclerotica verwachsen ist. Es ist nicht zu verwundern, dass eine solche Membran nicht die Functionen der Netzhaut ausüben kann, dass also ein grosser Defect im Gesichtsfelde stattfand. In anderen Fällen kann wie mehrfache Erfahrungen beweisen, der Zustand der Netzhaut anatomisch so wenig verändert sein, dass ihre Function keine Störung beobachten lässt.

In Bezug aut den Sehnerven, finde ich in den anatomischen Berichten über colobomatöse Augen keine Abnormität erwähnt; doch konnte Näheres über seine Beschaffenheit nicht ausgemacht werden. v. Ammon*) sah in dem Auge eines Schafsfötus ein Coloboma nervi optici, eine Spalte im Neurilem des Opticus, nachdem der Scleralspalt schon geschlosen war; auch bei neugebornen Kindern fand er zuweilen Klaffen der optischen Scheide. Arlt berichtet über einen Fall anato-

*) Archiv 1. Ophtb. IV. 1. pag. 41. 42. 
misch, Stellwag über einen andern ophthalmoskopisch, wo die Vortreibung der Selera die Insertionstheile zum Theile umfasste, wo also der Sehnerv die Grenze des Spalts bildete; dagegen ist mir kein Fall bekannt, wo, wie in dem obigen Falle, der Sehnerv sich mitten in dem staphylomatösen Theil der Sclera inserirte, cin Factum, das für die Entwickelungsgeschichte des Fötalspalts vielleicht von Belang ist. Ich erinnere in dieser Beziehung an die Beobachtung v. Ammon's*), dass in fötalen Augen zuweilen die Insertion des Sehnerven statt am hinteren Ende des Scleralspalts in dessen Mitte gefunden wird. Ich enthalte mich darauf hin eine $\mathrm{Hy}$ pothese aufzustellen, da nur die anatomische und histologische Untersuchung hier eine Entscheidung liefern kann.

*) Archiv f. Ophtb. IV, 1. p. 202. 